\title{
La creciente importancia de las relaciones económicas entre China y América Latina. El caso peruano
}

\author{
The growing importance of economic relations between China \\ and Latin America. The Peruvian case
}

\begin{abstract}
RESUMEN
Este artículo revisa los antecedentes de las relaciones comerciales de China con el mundo, el proceso de apertura que se dio a partir de la década de los setenta del Siglo XX y los profundos cambios que se vienen dando en las primeras décadas del Siglo XXI, con una guerra comercial entre Estados Unidos y China y con el avance que vienen teniendo la economía y las empresas chinas en sectores como energía y tecnología de comunicaciones. El artículo también revisa las relaciones comerciales y económicas entre China y los países de América Latina y el Caribe, poniendo un énfasis especial en el caso del Perú, donde China ya es el primer socio comercial tanto en exportaciones como en importaciones. Se señala la preocupación porque los países de la región están exportando a China fundamentalmente productos básicos como minerales y alimentos, por lo que es necesario lograr un intercambio más equitativo y con mayor valor agregado.
\end{abstract}

Palabras Clave: China; América Latina; Comercio Mundial; Economía Mundial; Perú.

\begin{abstract}
This article reviews the background of China's trade relations with the world, the process of opening up from the seventies of the twentieth century and the profound changes that have been taking place in the first decades of the twenty-first century, with a "trade war" between the United States and China and with the progress that the Chinese economy and companies have been having in sectors such as energy and technology communications. The article also reviews the trade and economic relations between China and the countries of Latin America and the Caribbean, placing special emphasis on the case of Peru, where China is already the leading trading partner in both exports and imports. There is concern that the countries of the region are mainly exporting to China commodities such as minerals and food, which is why it is necessary to achieve a more equitable and value-added exchange.
\end{abstract}

Keywords: China; Latin America; World Trade; World Economy; Peru.

\section{Enrique Javier Cornejo} Ramírez

ecornejor@unmsm.edu.pe ORCID: https://orcid.org/00000003-4683-9031

Universidad Nacional Mayor de San Marcos. Lima, Perú

Presentado: 29/06/2021 - Aceptado: 16/07/2021 - Publicado: 23/12/2021

(C) Los autores. Este artículo es publicado por la revista Gestión en el Tercer Milenio de la Facultad de Ciencias Administrativas Universidad Nacional Mayor de San Marcos. Este es un artículo de acceso abierto, distribuido bajo los términos de la licencia Creative Commons Atribución 4.0 Internacional (CC BY 4.0) [https://creativecommons.org/licenses/by/4.0/deed.es] que permite el uso, distribución y reproducción en cualquier medio, siempre que la obra original sea debidamente citada de su fuente original. 


\section{INTRODUCCIÓN}

Durante las dos primeras décadas del Siglo XXI, la presencia de China en América Latina ha sido creciente en términos de comercio, inversiones y préstamos, de manera que China se ha convertido en uno de los principales socios comerciales de la Región, incluso desplazando en algunos países a Estados Unidos en el natural liderazgo (por su tamaño y proximidad geográfica) que la economía norteamericana había mantenido a lo largo del Siglo XX.

¿Cuáles son las razones por las qué China ha incluido con prioridad a América Latina en los objetivos estratégicos de su política exterior? ¿Se trata de disputarle el liderazgo a Estados Unidos en su zona de influencia geográfica o responde a otros intereses estratégicos? ¿Cuáles son las características de la relación económica y comercial que China viene desarrollando con diferentes países de la Región? ¿Cuáles son los intereses de los países latinoamericanos para profundizar sus relaciones económicas con el gigante asiático? ¿Cuáles son las perspectivas de estas relaciones? ¿Cómo se está dando esta relación en el caso del Perú?

Estas son algunas de las interrogantes a las que trataremos de dar respuesta en este artículo.

\section{Los antecedentes de las relaciones eco- nómicas y comerciales de China con el mundo}

Los antecedentes de la reactivación de las relaciones económicas y comerciales de China con América Latina se remontan a la década del setenta en el Siglo XX. En aquellos años, Estados Unidos inició un acercamiento en sus relaciones diplomáticas y económicas con China, como parte de su estrategia para suavizar sus relaciones con la entonces Unión Soviética (URSS).

Las relaciones entre Estados Unidos y China se habían deteriorado hasta el extremo, cuando el Partido Comunista Chino ganó la guerra civil en 1949 y China participó en la guerra de Corea en 1950. Estados Unidos adoptó entonces la política deliberada de aislar a los gobernantes comunistas en Beijing. Pero en los años setenta, los objetivos estratégicos cambiaron por otras razones.
Como señala Kissinger (1994), exsecretario de Estado norteamericano, "a nuestro parecer, cuanto más se aplaza el enfrentamiento soviético con Occidente, más imposible resultaría la tarea de mantener unido al Imperio soviético, sobre todo porque a sus problemas políticos se les añadía el estancamiento económico" (p. 707).

China era clave para lograr este objetivo. El Departamento de Estado norteamericano trabajó entonces para recuperar la iniciativa diplomática en el contexto regional y mundial. Promover la apertura de China al mundo era una base fundamental de la política del presidente Richard Nixon, para contrarrestar el poderío y la influencia soviética.

Nixon y sus asesores sí lograron que las diversas partes de su política se apoyaran unas a otras. El enlace empezó a funcionar porque el gobierno de Nixon se las arregló para dar un gran incentivo a la moderación soviética, con su dramática apertura de China. (Kissinger, 1994, p. 712)

Como sucedió cuando terminó la Segunda Guerra Mundial, Estados Unidos, temeroso del avance político del otro vencedor del conflicto, la Unión Soviética, impulsó lo que se llamó la Guerra Fría, pero estimuló al mismo tiempo la rápida reconstrucción de Japón y Alemania con el Plan Marshall que inyectó importantes recursos financieros en las economías que antes eran adversarios en la guerra y entonces se convirtieron en socios políticos.

Pero lo que Estados Unidos no pudo prever es que esos aliados políticos pronto se convertirían en serios competidores comerciales que afectarían el déficit de su balanza comercial e incluso la libre convertibilidad Oro-US dólar con la que se constituyó el sistema monetario internacional de Bretton Woods en 1944.

De la misma manera, Estados Unidos no pudo prever, en los años setenta, que cincuenta años después, China se iba a convertir en un competidor comercial y económico de gran importancia, al punto que algunos analistas empiezan a ponerle fecha a una disputa por el liderazgo mundial en la que China resultaría siendo la nación vencedora. 
Diplomáticos norteamericanos como Kissinger estaban convencidos que excluir a China de las prioridades diplomáticas norteamericanas era un error. "En un sutil triángulo de relaciones entre Washington, Pekín y Moscú, mejoramos las posibilidades de acomodo con cada uno para aumentar nuestras opciones ante ambos" (Kissinger, 1994, p. 715), señalaba un discurso de Nelson Rockefeller cuando aspiró a la candidatura presidencial del Partido Republicano en 1968, una declaración que, por cierto, había redactado el mismo Kissinger.

Confirmando su política exterior, el presidente Nixon publicó un artículo en la Revista Foreign Affairs de octubre 1967:

Alargo plazo, sencillamente no podemos permitirnos dejar para siempre a China fuera de la familia de las naciones, para que alimente sus fantasías, agudice sus odios y amenace a sus vecinos. En este pequeño planeta no hay lugar para que mil millones del pueblo potencialmente más capaz, vivan en airado aislamiento. (Kissinger, 1994, p. 715)

Con la iniciativa norteamericana de transformar el mundo basado en dos potencias en un triángulo estratégico, los Estados Unidos anunciaron en 1969 una serie de decisiones unilaterales para mejorar su relación con China. Así, se levantó la prohibición de viajes de norteamericanos a China; se permitió a los norteamericanos llevar a su país artículos de procedencia china hasta por un valor de USS 100 dólares y se autorizaron embarques limitados de cereales a China.

Pero, lo más explícito del cambio de actitud de la diplomacia norteamericana con respecto a China, fue la advertencia que hizo el presidente Nixon a la Unión Soviética en el sentido que no verían con indiferencia un ataque soviético a China. Como bien lo advertía Kissinger (1994), "después de la apertura de China a los Estados Unidos, la Unión Soviética se encontró ante desafíos en dos frentes: la OTAN en el Oeste y China en el Este" (p. 724).

\section{La apertura de China al mundo}

Pero la apertura de China al mundo no solo puede ser explicada por el interés estratégico norteamericano. Un país continente y de historia milenaria como China no podía mantenerse en una posición de auto aislamiento económico y político. Así lo entendió ese extraordinario líder que se llamó Deng Xiaoping (1904-1997) que impulsó las reformas y la apertura entendiendo que este paso era fundamental para la propia existencia china.

Es a Deng a quien se deben los conceptos de un socialismo con características chinas incluido en la Constitución China en 1999, o el de Un país, dos sistemas y la famosa frase, conocida mundialmente: No importa el color del gato, lo importante es que cace ratones, que explica por sí sola el pragmatismo con que China afronta su actuación económica desde la época de Deng.

Dice Kissinger, con base a su experiencia personal en la diplomacia norteamericana con China: "Mao (Tse Tung), Zhou (Enlai) y después Deng (Xiaoping) eran personalidades extraordinarias. Mao era el visionario, el revolucionario implacable, sin piedad, a veces terrible; Zhou era el administrador elegante, encantador y brillante; y Deng era el reformador, de convicciones elementales" (Kissinger, 1994, p. 721).

Antonio Fernández Arce, periodista peruano que vivió y ejerció el periodismo cerca de cincuenta años en China, señala que para Deng. "Los estímulos materiales eran el motor del progreso humano (...) era un hábil negociador y genial administrador" (Fernández Arce, 2008, p. 149).

Es al pragmatismo de Deng Xiaoping que se debe la apertura de China al mundo como política de Estado, la implementación de las reformas para modernizar la economía china; la priorización del desarrollo económico sobre el tema político, así como la creación de las denominadas Zonas Económicas Especiales (ZEE) que han atraído a China miles de millones de US\$ dólares en capitales, tecnologías y nuevas formas de gestión.

La extraordinaria visión de Deng y su intuición sobre lo que más convenía a China, coinciden con lo que dice la literatura económica con respecto a cuáles son las razones por las cuales las empresas y las naciones tienen que lograr una mayor y mejor inserción en el escenario internacional. 
Adam Smith (1776) señalaba tempranamente la importancia de la división del trabajo y destacaba las limitaciones del mercado interno para seguir aprovechando las ventajas que brinda la especialización. La participación activa de un país en el comercio internacional responde a necesidades básicas del crecimiento y diversificación que necesitan tener las empresas en un contexto local que ya no les permite mejores rendimientos.

Sostiene Krugman et al. (2012) que "cuando hay economías de escala externas, el comercio internacional hace posible concentrar la producción mundial en una única localización $\mathrm{y}$, por lo tanto, reducir los costos aprovechando las ventajas de unas economías externas aún más potentes" (p. 147)

De modo que hay poderosas razones económicas, considerando a todos los autores citados que explican la necesidad de que un país-continente como China tenga un papel cada vez más importante en la economía mundial.

A las razones políticas y económicas, habría que agregar las razones de tipo cultural que una nación con historia milenaria y cultura tan diversa como China no pude dejar de considerar en su relacionamiento con el mundo.

China inició entonces una participación muy activa en los principales organismos multilaterales, empezando por la Organización de las Naciones Unidas (ONU), institución en la que participa desde su fundación y en la que hoy tiene un papel protagónico en el Consejo de Seguridad, siendo uno de los cinco países permanentes y con poder de veto, junto con Francia, Rusia, el Reino Unido y los Estados Unidos.

A la Organización Mundial del Comercio (OMC), institución creada en 1994 en el marco del Acuerdo General sobre Aranceles Aduaneros y Comercio (GATT), por el Acuerdo de Marrakech, China ingresó en diciembre de 2001 y en apenas una década de participación activa en las negociaciones multilaterales de comercio y en acuerdos bilaterales y regionales en diversas partes del mundo, China es actualmente principal protagonista de las exportaciones e importaciones mundiales de bienes y servicios.
Como lo señalamos en una publicación que hicimos hace algunos años, desde 2010, es decir, desde hace más de una década, China ya es la primera potencia comercial en el mundo. ¿Cómo llegamos a esta conclusión? En ese año, si sumamos la participación de China $(8,8 \%)$ y de Hong Kong $(2,6 \%)$ en el total del comercio mundial, ambos representaban el 11,4 \% de participación en el comercio global, superando el $11,1 \%$ de Estados Unidos.

Por otro lado, en los inicios de la tercera década del Siglo XXI, China se mostraba ya como la segunda potencia mundial en términos productivos, después de Estados Unidos. Esa posición la tiene China desde el segundo trimestre de 2010, período en el que pudo superar el valor del PBI de Japón cuya economía estaba entonces agobiada por un complejo proceso deflacionario.

China es el país más poblado del mundo con 1,433 millones de habitantes; es el cuarto país con mayor extensión territorial del mundo con 9,597 kilómetros cuadrados (después de Rusia, Canadá y Estados Unidos); China es el primer consumidor de energía del mundo y pronto será el mayor ofertante de energía no convencional. Son cifras espectaculares que hablan por sí mismas.

Teniendo en cuenta las condiciones de especialización que plantea la teoría del comercio internacional, muchos tienen la idea equivocada de que China se ha especializado solo en productos relativamente baratos orientados hacia mercados masivos de consumo en el mundo. Esa concepción es errónea.

Si bien este tipo de especialización estuvo presente en décadas anteriores, cada vez es más evidente que las industrias fuertemente intensivas en capital y tecnología forman parte de la oferta exportable china en diversos sectores; en algunos de ellos -como es el caso -por ejemplo- del transporte público con energía eléctrica, China ya es indiscutiblemente el líder mundial. Igual sucede en otros sectores de alta tecnología e innovación como es el caso de la tecnología $5 \mathrm{G}$ en la telefonía móvil.

Cuando en 2009, el gobierno peruano evaluaba qué tipo de tecnología iba a adoptar para implementar la televisión digital en el país, se eligió el estándar digital de origen japonés (IS- 
DB-T), pero el segundo en la calificación fue la correspondiente tecnología china, muy por encima de los estándares europeo y norteamericano.

Pero ello no ha ocurrido de un momento a otro. Ha sido producto de un proceso que se está dando desde hace varias décadas. En el contexto de este proceso se han dado cambios importantes al interior de la economía china en busca de menores costos, mayor eficiencia y productividad.

En las últimas décadas, en China se evidencia un proceso de relocalización productiva, especialización y aprovechamiento de sinergias entre macro ciudades o macro circuitos económicos. Así, Beijing -la ciudad capital- se ha especializado en actividades de comercio, publicidad, bienes raíces, cultura y turismo, servicios informáticos; Shanghái -por su parte- lo ha hecho en semiconductores, circuitos integrados, inteligencia comercial, retail, logística y cadena de suministro (supply chain).

De otro lado, Shenzhen se ha especializado en robótica, diseño y equipamiento de manufactura, tecnología avanzada; la ciudad de Hangzhou se ha especializado en la producción y exportación de textiles, servicios, e-commerce, turismo y ecología; mientras que las ciudades de Foshan y Tangshan se han especializado en la producción de cerámica.

Además de esta especialización sectorial distribuida geográficamente en las principales ciudades del país, se han formado también macro ciudades o macro círculos económicos, en busca de mayor eficiencia y productividad, tales como la Zona Económica Beijing- Tianjin-Hebei o la Zona económica triangular del Río Yangtze.

Importancia especial para lograr estos avances han tenido las denominadas Zonas Económicas Especiales (ZEE), creadas a partir de la década del ochenta. Existen diversos tipos de ZEE, desde las destinadas a atraer inversiones extranjeras para el desarrollo económico y tecnológico (tales como Shenzhen, Zhuhai, Xiamen o Pudong en Shanghai), hasta las zonas de desarrollo de alta tecnología. En todas ellas se ofrece adecuada infraestructura, incentivos fiscales, así como facilitación de licencias y otros servicios.
En las principales ciudades chinas se han ubicado y se siguen ubicando -y en este sentido toma vigor la teoría de Krugman sobre las economías externas de escala y su relación con el comercio internacional- las principales empresas transnacionales chinas, varias de las cuales ya se ubican en los primeros lugares del ranking mundial de Fortune500.

En 2019, según el ranking publicado por esta prestigiosa revista internacional, por primera vez China superó en número de empresas a los Estados Unidos en el ranking de las 500 empresas más valoradas del mundo (Fortune500, 2020).

Entre las primeras 50 empresas del Ranking Global de Fortune 500, se encuentran trece compañías chinas pertenecientes a los sectores, petrolero, petroquímico, construcción, telefonía, producción electrónica, servicios de ingeniería, banca y seguros. Toda una diversificación que hay que tener en cuenta y que seguirá sorprendiendo en los próximos años.

Aun cuando Estados Unidos sigue dominando el sector tecnológico, con líderes mundiales como Apple, Amazon, Google, Microsoft y Facebook, entre otros, la presencia de empresas chinas del área tecnológica será creciente en los próximos años.

China, de otro lado, viene desarrollando mega planes de infraestructura como la denominada "Nueva Ruta de la Seda" que incluye la construcción de puertos, líneas ferrocarrileras, oleoductos, gaseoductos en cinco continentes, lo que sin duda tendrá un gran impacto de China en la economía global.

\section{La denominada guerra comercial entre China y los Estados Unidos}

Así como en los años setenta y ochenta del Siglo $\mathrm{XX}$, se produjo una guerra comercial entre Estados Unidos frente a Alemania, Japón y otros países, guerra en la que -como ya hemos señalado- no salió muy bien librada la economía norteamericana, en la tercera década del Siglo XXI el mundo presencia una nueva "guerra comercial", esta vez entre Estados Unidos y China, y nuevamente los resultados no parecen ser tan favorables a los Estados Unidos. Además, como 
elemento diferencial, esta "guerra" se da en un contexto de interdependencia global.

El tema de fondo en esta nueva versión de la guerra económica y comercial es: ¿quién va a liderar en el próximo ciclo económico? Lo que resulta ya evidente es que el ciclo basado en el petróleo y derivados está acabando (autos, barcos, aviones y otras industrias claves en el Siglo XX han estado basados en el petróleo como principal fuente de energía). Pero, el nuevo ciclo productivo y tecnológico tiene que ver con la generación de energías limpias, y China se viene preparando hace dos décadas para ello.

Sin embargo, los Estados Unidos pareciera que va en sentido contrario. Un documento que resume la política estratégica de la Casa Blanca publicado en 2017 señalaba que "el petróleo y las energías fósiles son la base de la energía futura". Es posible que con la Administración Biden cambie en algo esta posición, sobre todo volviendo a poner a Estados Unidos en la agenda y compromisos multilaterales que había abandonado deliberadamente la Administración Trump pero, es evidente que Estados Unidos ya está retrasado frente a China y otros países en esta carrera por la energía limpia.

Los adelantos tecnológicos que se vienen desarrollando en China en este campo permiten incrementar la productividad y ahorrar energía. Estados Unidos, por su parte, no ha podido competir eficazmente en estas nuevas tecnologías. "Ha llegado tarde y ha llegado caro" (Enrique Cornejo Digital, 2020).

Son conocidos los problemas de productividad y competitividad de la economía norteamericana. Así, la tecnología de celulares 5G ya está en el mercado y producirá cambios espectaculares: mucha mayor velocidad, mayores interacciones, menores costos y mayor duración de la batería, entre otras ventajas. Y la empresa china Huawei es la líder en esta tecnología.

En agosto de 2018, Huawei lanzó su Red 5G. Por su parte, ATT, la empresa norteamericana, había programado lanzar su Red 5G en diciembre de 2018, pero se atrasó y solo lo hizo parcialmente. La estrategia de Estados Unidos ha sido entonces la de sabotear la entrada de Huawei a ese país e intentar influir para que tampoco pueda entrar en otros países de Occidente, pero lo cierto es que muchos países están comprando la Red 5G china. Así, el boicot norteamericano a Huawei no está cumpliendo con los objetivos que se había propuesto la política exterior norteamericana.

Por otro lado, China ya es líder mundial en la producción de paneles solares. En el año 2000, China empezó a producir partes y piezas para paneles solares compitiendo con los europeos que eran los que empezaron en esa línea de producción. Desde entonces, los chinos se han especializado, se han hecho más eficientes y han disminuido los costos, de manera que un panel solar, hacia el año 2021, cuesta la sexta parte de lo que costaba hace veinte años y es de procedencia china.

También China destaca mundialmente en la producción mundial de autos eléctricos, así como de buses eléctricos para el transporte público. En los primeros años del nuevo siglo, China empezó a impulsar la fabricación de buses eléctricos que se están adquiriendo en diversas ciudades del mundo para tener un transporte público que no contamine el aire $y$, además, avanzar en el cumplimiento de los compromisos mundiales para contrarrestar el cambio climático.

Diversos países del mundo, como por ejemplo los de la Unión Europea, han puesto metas y fechas muy precisas para sustituir el parque automotor basado en Diesel o gasolina y cambiarlo por autos eléctricos. Acuerdos mundiales como los de París 2015 van en el mismo sentido.

Para avanzar en este propósito y convertirse en uno de los principales proveedores de autos y buses eléctricos en el mundo, China ha realizado alianzas estratégicas con empresas alemanas de autos. Está en proceso un acuerdo con Bolivia para producir baterías de litio, aprovechando los yacimientos de ese metal existentes en el país andino. China lidera ya el impulso al cambio en la matriz energética mundial y el comercio energético en el planeta, superando también en este sector a los Estados Unidos.

Existen otros sectores productivos y de servicios en los que China viene teniendo creciente participación en el comercio mundial y ocupa cada vez mayores espacios de liderazgo, como son, entre otros, los sectores de minería, construcción, naviero y de infraestructura 
ferrocarrilera y portuaria. Baste decir que la empresa china Hutchison Port Holdings es la líder mundial en operaciones portuarias.

La pregunta que surge es ¿por qué los Estados Unidos están perdiendo en varios sectores esta "guerra económica y comercial" con China? El propio Krugman, en una entrevista para BBC News Mundo (2020), señalaba que el verdadero problema de la competitividad para la economía norteamericana radicaba en la posibilidad de que la competencia internacional excluya a Estados Unidos de la competencia internacional, incluso en algunas industrias donde estas deberían tener ventajas comparativas.

Para Krugman, en una entrevista para BBC News Mundo (2020), la pérdida de competitividad de muchas industrias manufactureras en los Estados Unidos se debe a tres principales razones: la baja tasa del ahorro nacional; los modestos gastos en investigación y desarrollo comparados con los que se dedican a este concepto en otros países; y, la baja calidad de la educación básica.

Sobre lo que sucederá en las próximas décadas con los liderazgos de China y de Estados Unidos hay diferentes posiciones. Para autores como Krugman, Estados Unidos es un país altamente productivo, con gran diversidad y creatividad y esos factores seguirán siendo decisivos. Paul Krugman, el premio Nobel de Economía sostiene que "China tiene una gran fortaleza, pero no puede ser un líder mundial. Ser un gran poder no es suficiente para tener liderazgo mundial; necesitas que el mundo crea en ti" (BBC News Mundo, 2020).

Por otro lado, para el economista peruano Oscar Ugarteche, Estados Unidos tiene un serio problema de productividad y competitividad; sus costos laborales domésticos son muy altos, menciona que "a Estados Unidos le está pasando lo mismo que al Reino Unido en 1920: está en proceso de declive tecnológico, proceso que es lento, pero 'se lleva' ciudades enteras" (Enrique Cornejo Digital, 2020).

China está desarrollando importantes esfuerzos para seguir mejorando la eficiencia y productividad de su mercado interno de manera de lograr, a su vez, un mejor posicionamiento en el escenario global, pero esta no es una tarea fácil y requiere asumir varios desafíos que su actual líder Xi Jinping parece tener muy claros.

El yuan no es una moneda global. Para tener esta característica China debería tener mercados financieros abiertos (con libertad para entrar y salir) y lograr credibilidad para que sus obligaciones financieras sean respetadas. China tiene un mercado financiero doméstico poco desarrollado y con controles de capitales. Elevar la productividad de su sector agrícola es otro gran desafío para la economía china.

Por otra parte, China ha demostrado tener la capacidad de hacer políticas públicas efectivas y es un país grande, pero también es un país autocrático en muchos aspectos. Aun cuando su pragmatismo económico lo ha acercado al mundo, sus prácticas poco democráticas y la falta de transparencia en la manera cómo funcionan sus mercados domésticos le generan dificultades en sus relaciones con las principales economías del mundo, especialmente de Occidente.

Lo que nadie duda es de la gran capacidad e influencia que China ya tiene en las decisiones estratégicas a nivel mundial. La propia pandemia del Coronavirus demostró, de un lado, sus debilidades, pero -de otro lado, evidenció su capacidad de reacción rápida y eficiente para controlar la crisis sanitaria.

Si consideramos los doce pilares que explican el Índice Global de Competitividad que elabora anualmente el World Economic Forum (WEF), China todavía se encuentra entre los primeros treinta países del mundo (aunque Hong Kong se ubica entre los diez primeros países del ranking).

Sin embargo, si consideramos las innovaciones tecnológicas que tendrán -según los especialistas- un mayor impacto en el mundo en los próximos años (como, por ejemplo, la automatización del conocimiento, el Internet de las cosas, la impresión 3D, los avances en la genómica y en la robótica, así como la energía renovable) muchas empresas chinas ya empiezan a destacar e incluso a liderar estas innovaciones.

De manera que lo que se puede esperar en los próximos años es una economía china cada vez más competitiva y con mayor presencia e influencia en la economía mundial. Por lo pronto, la 
crisis sanitaria de 2020 impactó negativamente en prácticamente todas las economías del mundo que registraron tasas negativas en su producto bruto interno (PBI), excepto en el caso de China cuya economía creció en 1 por ciento en 2020 y se estima que crecerá más de 8\% en 2021.

\section{Las relaciones económicas y comercia- les de China con América Latina}

Son varias las razones estratégicas por las cuales China ha decidido profundizar sus relaciones económicas y comerciales con América Latina. China está en búsqueda de fortalecer su presencia económica y comercial en todo el mundo, lo que implica buscar nuevos socios.

En el 2008, el comercio total de China con América latina ascendía a US\$ 143 mil millones; una década después casi se había duplicado. Mayor crecimiento se observa en las cifras de inversión directa china en América Latina que creció de US\$ 30 mil millones en 2010 a más de US\$ 200 mil millones en 2017; la mayoría de esas inversiones están relacionadas al sector minero, energético, alimenticio e infraestructura.

El propio presidente de China, Xi Jinping, en enero de 2015, en Beijing, con ocasión de la Primera Reunión Ministerial del Foro de la Comunidad de Estados Latinoamericanos y Caribeños $(\text { CELAC })^{1}$, señalaba claramente la estrategia de su país sobre lo que denominaba "Escribir juntos un Nuevo Capítulo de la Asociación de Cooperación Integral China-América Latina y el Caribe" (Xi Jinping, 2015).

Llegó el momento oportuno para el progreso conjunto y la profundización de la cooperación entre China y América Latina y el Caribe. China y los países latinoamericanos y caribeños representan juntos una quinta parte de la superficie del planeta, una tercera parte de la población mundial y una octava parte de la economía global, lo que proporciona enormes ventajas y valiosos recursos para el pleno desarrollo de las relaciones entre China y la región hacia una

1 La Comunidad de Estados Latinoamericanos y Caribeños (CELAC) es un mecanismo intergubernamental de diálogo y concertación política que fue creado en diciembre de 2011 y lo integran treinta y tres países de la región. Como parte de sus tareas, el CELAC participa en diálogos con la Unión Europea, China, Rusia, Corea, los Estados Árabes del Golfo, Turquía y Japón. mayor profundidad. (...) trabajaremos juntos por realizar la meta de que, en los próximos diez años, el intercambio comercial entre China y la región llegue a los 500 mil millones de dólares y el stock de la inversión directa china en la región, a los 250 mil millones de dólares. (Xi Jinping, 2015)

La estrategia de Xi Jinping incluye el criterio $(1+3+6)$, que consiste en un plan (de cooperación entre China y la Región), tres motores (Comercio, Inversiones y Finanzas) y seis campos de acción (energía, infraestructura, agricultura, manufactura, Innovación y tecnologías de la información), tal como se observa en la Figura 1.

No cabe duda de que China ve en la región latinoamericana un socio estratégico en dos sentidos: el primero relacionado a su gran disponibilidad de productos básicos (minerales, alimentos), y, el segundo, vinculado a la necesidad de encontrar en la región mercados para la cada vez más creciente oferta china de manufacturas y tecnologías. América Latina, cuenta -a principios de la tercera década del nuevo siglo- con una población de aproximadamente 640 millones de habitantes y un producto bruto interno estimado en US\$ 5.8 billones.

La pregunta que surge es si este relacionamiento, que se ha profundizado en las primeras dos décadas del Siglo XXI, va a considerar también a mediano plazo asociaciones entre gobiernos o entre empresas de China y América Latina para el desarrollo conjunto de proyectos destinados a elaborar bienes y servicios con mayor valor agregado, pues -como señalan-varios autores, lo que se aprecia en estos tiempos es más bien un incentivo a la reprimarización de la región.

En algunos países de la región se observa más que en otros esta predominancia de unos cuantos productos básicos de exportación explicando el mayor valor exportado a China. Así, por ejemplo. Brasil exporta a China más del 60 por ciento de su producción de soya; Argentina exporta a China el 80 por ciento de su producción de soya y Chile el 80 por ciento de su producción de cobre.

Para muchos de los países latinoamericanos, incrementar la cantidad y calidad del 
Figura 1

"1+3+6": Estrategia China para América Latina

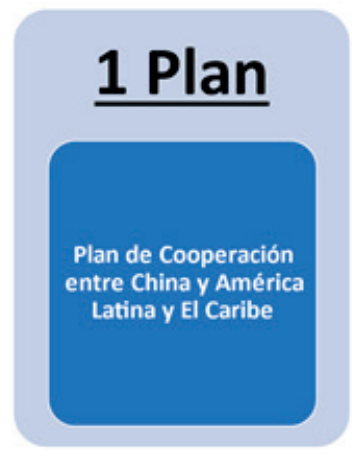

Nota. Elaboración propia.
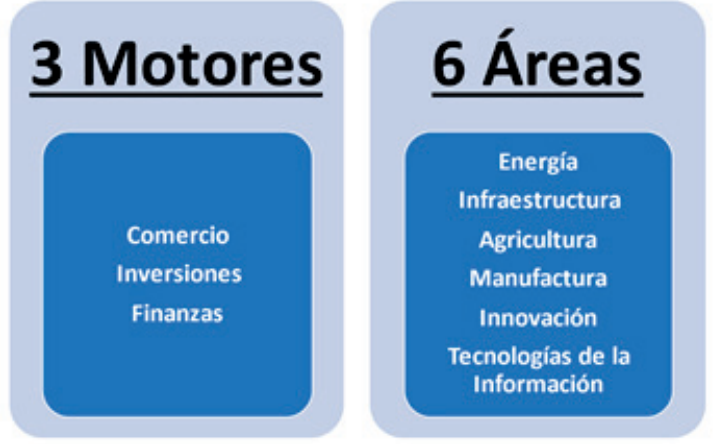

intercambio comercial y de la cooperación económica con China, no es solo negociar con el mercado más grande del planeta y un país que viene avanzando en su liderazgo mundial, es también una oportunidad de diversificar su comercio internacional y su relacionamiento estratégico con el mundo.

Bartesaghi (2019) encuentra que, en 2018, ocho grupos de productos primarios explican el 90 por ciento de las exportaciones de América Latina a China, lo que evidencia una alta concentración en este tipo de productos. Minerales metalíferos explican el 29\% del total; semillas y frutos oleaginosos (incluida la soya) explican el 24\%; combustibles y minerales el 16\%; cobre y sus manufacturas el 10\%; pasta de madera el $5 \%$; carnes el 3\%; pescados y crustáceos el 1\%; residuos y desperdicios de las industrias alimentarias el $1 \%$.

Por su parte, Durán Lima y Pellandra (2017), en un documento publicado por la Comisión Económica para América Latina (CEPAL), señalan al respecto:

En casi todos los países de América Latina la estructura exportadora ha mostrado conjuntamente un aumento en el peso de las materias primas y de la manufactura basada en recursos naturales, que representan en 2014 cerca del 60\% de las exportaciones de América Latina y el Caribe, con la excepción de México. (...) si se considera únicamente el cambio en la estructura exportadora de cada país a China, se observa un aumento aún más dramático del peso de las materias primas y sus manufacturas, las que en varios países superan el 90\% del total, y en México llegan al $46 \%$. (p. 17)

Aviles Quintanar y Wong González (2019) concluyen que sí se produce este efecto "reprimarizador", entendiendo como tal el retorno de un país al sector primario de su economía.

Conforme a la muestra de 20 países de la región (...) solamente Brasil representa un tercio del PBI latinoamericano (...), Argentina un $10 \%$, Chile un 5\%, Perú $4 \%$, Ecuador $2 \%$ y Uruguay el uno por ciento. En su conjunto, este bloque de países que están siendo influenciados por China en el proceso reprimarizador, representan el 55\% del PBI de América Latina. (Aviles Quintanar y Wong González, 2019, p. 120)

No se observa, por el momento, al menos explícitamente, un interés de China de disputar el liderazgo estratégico a Estados Unidos en América Latina, aun cuando -como estamos señalando- ya se ha producido en la práctica una superación en el tema comercial en el caso de varios países de la región. Parecería ser que lo que interesa más a la estrategia china son sus intereses económicos y comerciales antes que los políticos. Pero esta situación podría variar más adelante.

Sobre este último punto debe señalarse que Estados Unidos ha descuidado por un buen 
tiempo la calidad de sus relaciones con América Latina. Las tres iniciativas norteamericanas para lograr acuerdos de alcance hemisférico han fracasado. Primero fue la Alianza para el Progreso que se originó en la década de los años sesenta del Siglo XX y que se frustró con el asesinato del presidente Kennedy; después se dio la Iniciativa de las Américas, promovida por el presidente George H.W. Bush en junio de 1990 que tuvo alcances limitados; $y$, finalmente el evidente fracaso de la denominada "Área de Libre Comercio para las Américas-ALCA" que impulsó el presidente Bill Clinton y que tuvo a Brasil y otros países de la Región como sus principales detractores.

Estados Unidos ha tratado entonces de compensar el fracaso de estas iniciativas con el establecimiento de acuerdos bilaterales de libre comercio con los países centroamericanos, los países del Caribe, Chile, Perú y Colombia, mientras que con México y Canadá mantiene un acuerdo trilateral de libre comercio. Pero, el reto de una integración hemisférica más profunda sigue pendiente y esto puede ser un campo propicio para el avance de China en el desarrollo de sus intereses en la región. De hecho, China también está impulsando últimamente tratados de libre comercio o de asociación estratégica con los países de la región.

Pero ¿cuáles son las razones por las que China está desarrollando esta agresiva política de penetración en América Latina?

López Villafañe (2018) identifica seis razones principales por las cuales China prioriza esta relación:

los factores que vinculan a China con Latinoamérica se resumen en los siguientes planes: 1) comprar materias primas en la región, 2) realizar inversiones para la producción de recursos naturales y de infraestructura, 3) exportar manufacturas, 4) propiciar la estabilidad regional, 5) fomentar la multipolaridad para reducir el poder de Estados Unidos, y 6) eliminar a Taiwán como rival. (p. 17)

Las relaciones económicas y comerciales entre China y América Latina también tienen que evaluarse en la competencia que los productos chinos tienen con los productos latinoamericanos en terceros mercados. Es el caso, por ejemplo, de los textiles y confecciones chinas que, desde 2005 ingresan al mercado norteamericano sin las tradicionales cuotas que ese país ponía a importaciones de este sector provenientes de diferentes países.

\section{Las relaciones económicas y comercia- les de China con el Perú}

China y Perú pertenecen a ese pequeño y privilegiado grupo que pueden considerarse como países-civilización. La historia de China nos refiere a documentos escritos hace cerca de 3,500 años, sin embargo, fuentes orales se refieren a una historia que ya tiene más de 5,000 años. En el caso peruano, con los estudios recientes que se están haciendo con respecto a la Civilización de Caral (que se ubicó a cerca de 200 kilómetros al norte de Lima), podemos referirnos a que la civilización peruana tiene ya más de 5,000 años de antigüedad.

Hay, por lo tanto, entre los dos países-civilización aspectos históricos y culturales que los unen, más allá de los temas económicos, comerciales o incluso políticos. A fines de la década del cuarenta del Siglo XIX, se produjo la primera inmigración de culíes chinos al Perú y en la segunda mitad del Siglo XX, en noviembre de 1971, se anunció oficialmente el inicio de las relaciones diplomáticas entre Beijing y Lima.

Ya en el nuevo contexto, las primeras operaciones comerciales se relacionaron con la exportación de harina de pescado del Perú hacia China. Como se conoce, el Perú es el primer país productor mundial de harina de pescado, producto que es utilizado como parte del alimento balanceado para el ganado. China se convirtió desde entonces en el primer comprador de harina de pescado de origen peruano. También se exportó, en esos primeros tiempos de relaciones diplomáticas entre ambos países, productos mineros como cobre, plomo y zinc.

Ese año (1971) fueron vendidas al gran mercado chino las primeras ciento cincuenta mil toneladas de harina de pescado, veinte mil de aceite de pescado, cuarenta mil de cobre, diez mil de plomo, diez mil de zinc. Se negoció una primera línea de crédito china por más de 
40.000.000 de dólares sin intereses, con diez años de gracia y se obtuvo para el Perú el trato de nación más favorecida. (Fernández Arce, 2008, p. 434)

En la Tabla 1 podemos observar cómo ha evolucionado la estructura de exportaciones del Perú considerando los diez principales mercados de destino en un período de siete décadas que va desde 1950 hasta 2018.

A mediados del Siglo XX, claramente el principal mercado para las exportaciones peruanas era la economía norteamericana, que solo representaba la cuarta parte del total de dichas exportaciones. El segundo mercado importante para las exportaciones procedentes del Perú era América Latina y el tercer mercado más importante era el europeo.

Veinte años después, en 1970, Estados Unidos había consolidado su liderazgo en América Latina y en Perú representaba la tercera parte de las exportaciones peruanas. Japón, Alemania y otros países europeos estaban entre los primeros mercados de destino de dichas exportaciones.

A principios del Siglo XXI, todavía los Estados Unidos era el principal socio comercial del Perú (27 por ciento de las exportaciones totales), pero se observaba ya la presencia de China como el cuarto mercado de destino de las exportaciones peruanas, con el 6 por ciento de sus exportaciones totales.

En el año 2010, China ya era el segundo mercado en importancia para las exportaciones peruanas después de Estados Unidos (16 por ciento versus 27 por ciento de las exportaciones totales, respectivamente). En el año 2018, China pasó a constituirse en el primer socio comercial del Perú, explicando el 27 por ciento del total exportado por el Perú, frente al 16 por ciento de los Estados Unidos.

Ese año, entre los diez principales mercados de destino para las exportaciones peruanas, había cinco países asiáticos (41 por ciento del total exportado), Estados Unidos (16 por ciento del total), tres países europeos ( 9 por ciento del total) y Chile (2 por ciento del total). Esta composición responde a una estrategia de largo plazo del Estado peruano que, en dos décadas, negoció, suscribió y ratificó un total de 22 acuerdos comerciales tipo tratados de libre comercio, incluyendo un Acuerdo de Asociación Estratégica con la República Popular China.

En la Tabla 2 se observa la estructura de las importaciones peruanas por país de origen entre 1950 y 2018. En 1950, Estados Unidos explicaba prácticamente el 50 por ciento del total de importaciones peruanas; los países europeos (integrantes de la Comunidad Económica

Tabla 1

Principales destinos de exportaciones peruanas: 1950-2018 (Como porcentajes del total de exportaciones al mundo)

\begin{tabular}{ccccc}
\hline Orden/Años & $\mathbf{1 9 5 0}$ & $\mathbf{1 9 7 0}$ & $\mathbf{2 0 0 0}$ & $\mathbf{2 0 1 8}$ \\
\hline 1 & EEUU (25.1) & EEUU (33.1) & EEUU 27.0) & China (27.0) \\
2 & ALALC (26.4) & Alem (15.2) & Suiza (8.0) & EEUU (16.0) \\
3 & CEE (15.0) & Japón (13.6) & U.K. (8.0) & India (5.0) \\
4 & EO (13.9) & P. Bajos (9.6) & China (6.0) & Corea (5.0) \\
5 & R EU (6.0) & Bélgica (4.6) & Japón (4.0) & Suiza (4.0) \\
6 & R AL (5.3) & U.K. (2.5) & Chile (4.0) & Japón (4.0) \\
7 & Asia (4.9) & España (2.3) & Brasil (3.0) & Brasil (3.0) \\
8 & Canadá (2.8) & Francia (1.8) & México (2.0) & P. Bajos (2.0) \\
9 & R M (0.4) & México (1.3) & España (2.0) & Chile (2.0) \\
\hline 10 & Ocean (0.2) & Col (0.9) & Col (2.0) & 71
\end{tabular}

Nota. REU: Resto de Europa, RAL: Resto de América Latina, RM: Resto del Mundo, Ocean: Oceanía, P. Bajos: Países Bajos, ALALC: Asociación Latinoamericana de Libre Comercio, U.K: United Kingdom, EO: Europa Oriental. Adaptado de "World Integrated Trade Solution", por Banco Mundial, 2021 (https://wits.worldbank.org/CountryProfile/es/Country/PER/StartYear/1992/EndYear/2018/TradeFlow/ ExportPartener/BY-COUNTRY/Indicator/XPRT-PRTNR-SHR). Copyright 2021 de la The World Bank Group. 
Tabla 2

Importaciones Peruanas por principales países de origen: 1950-2018 (como porcentajes del total de exportaciones al mundo)

\begin{tabular}{|c|c|c|c|c|}
\hline Orden/Años & 1950 & 1970 & 2000 & 2018 \\
\hline 1 & EEUU (49.6) & EEUU (31.9) & EEUU (23.0) & China (23.0) \\
\hline 2 & Canadá (5.5) & Alemania (12.1) & Venez (8.0) & EEUU (21.0) \\
\hline 3 & ALALC (7.9) & Japón (7.9) & Japón (6.0) & Brasil (5.0) \\
\hline 4 & R. AL (0.3) & Argent (6.4) & Colomb (5.0) & México (4.0) \\
\hline 5 & CEE (9.3) & Canadá (4.5) & Chile (5.0) & Ecuador (4.0) \\
\hline 6 & EO (6.8) & Colomb (4.3) & Brasil (5.0) & Colomb (3.0) \\
\hline 7 & R Eu (15.8) & U.K. (4.3) & Ecuador (4.0) & Chile (3.0) \\
\hline 8 & Asia (1.9) & Italia (2.9) & Argent (4.0) & Japón (2.0) \\
\hline 9 & Oceanía (0.2) & Bélgica (2.4) & México (3.0) & India (2.0) \\
\hline 10 & RM (2.8) & P. Bajos (2.1) & China (3.0) & España (2.0) \\
\hline$\%$ Acumulado & 100 & 78.8 & 66 & 69 \\
\hline
\end{tabular}

Nota. REU: Resto de Europa, RAL: Resto de América Latina, RM: Resto del Mundo, P. Bajos: Países Bajos, ALALC: Asociación Latinoamericana de Libre Comercio, U.K: United Kingdom, EO: Europa Oriental. Adaptado de "World Integrated Trade Solution", por Banco Mundial, 2021 (https://wits.worldbank.org/CountryProfile/es/Country/PER/StartYear/1992/EndYear/2018/TradeFlow/ExportPartener/ BY-COUNTRY/Indicator/XPRT-PRTNR-SHR). Copyright 2021 de la The World Bank Group.

Europea-CEE Europa Oriental y el resto de Europa) representaban el 32 por ciento de dicho total; mientras que los países latinoamericanos, integrantes de la Asociación Latinoamericana de Libre Comercio-ALALC, explicaban el 8 por ciento del total importado por el Perú.

En el año 2000, Estados Unidos seguía liderando la importación de productos peruanos, aunque con una participación mucho menor que cinco décadas atrás, con un 23 por ciento del total. Como sucedió en el caso de las exportaciones, China ya aparece en ese año entre los diez primeros países de origen de las importaciones del Perú, con un 3 por ciento del total.

El año 2018, China ya era el primer socio comercial del Perú también en lo que respecta a importaciones con un 23 por ciento del total, desplazando a Estados Unidos que ese año solo representó el 21 por ciento. Completan el cuadro de los diez primeros importadores del Perú cinco países latinoamericanos (19 por ciento del total), dos países asiáticos (4 por ciento del total) y España (2 por ciento del total).

Como anotan Durán Lima y Pellandra (2017), la incidencia de las importaciones provenientes de China se incrementó desde 0.9\% en 2000 a $5.5 \%$ en 2012, es decir, se sextuplicó, principalmente en equipos electrónicos y ópticos, maquinarias y equipos, automotores y sus partes, así como textiles, confecciones y calzado. Este intercambio comercial entre Perú y China se ha facilitado a partir de la vigencia del Acuerdo de Asociación Estratégica suscrito entre ambos países en 2009 y que entró en vigencia en 2010.

La relación comercial bilateral es creciente, aunque deficitaria para Perú. Las exportaciones peruanas consisten principalmente en productos mineros, pesqueros y agroindustriales. Las exportaciones chinas son principalmente maquinaria y equipo, textiles y confecciones, automotores y sus partes y químicos. Esta estructura comercial debe mejorar cualitativamente en los próximos años, pero todavía se muestra con alta incidencia de productos primarios de exportación por parte del Perú, confirmando lo que se aprecia a nivel latinoamericano.

\section{CONCLUSIONES}

En este artículo hemos podido apreciar la importancia que para China tiene profundizar sus relaciones con el mundo por razones económicas, estratégicas y culturales. Como resultado de esta apertura al mundo la presencia económica y comercial de China ya se hace notar y, en algunos sectores, está avanzando sustantivamente hacia el liderazgo mundial o ya lo logró, 
como es el caso de las tecnologías vinculadas a energías limpias o el caso de la tecnología 5G en la telefonía móvil.

China ya es la primera potencia comercial del planeta y la segunda potencia productiva mundial después de Estados Unidos. La denominada "guerra comercial" entre Estados Unidos y China, no parece estar favoreciendo a la economía norteamericana.

A diferencia de guerras comerciales anteriores entre Estados Unidos, Japón y Alemania, la actual se da en un contexto de globalización y con un actor protagónico-China- que no se subordina políticamente a Estados Unidos, como si lo hicieron Japón y Alemania. Sin embargo y por el momento, a China parece interesarle más avanzar en sus objetivos económicos y comerciales que en los estratégicos para disputarle el liderazgo mundial a Estados Unidos.

La relación entre China y América Latina se ha profundizado en las últimas décadas, con una serie de iniciativas en los ámbitos comercial, financiero y de inversiones. Sin embargo, la estructura comercial de esta relación está enfatizando en la exportación desde la Región de productos básicos, como minerales, energía y alimentos, lo que -a decir de varios autores- estaría llevando a un proceso de reprimarización en América Latina.

En el caso del Perú, China ya es su primer socio comercial tanto en exportaciones como importaciones. La suscripción en 2009 de un Acuerdo de Asociación Estratégica entre ambos países ha contribuido a profundizar la relación comercial y de inversiones. Sin embargo, la estructura comercial todavía muestra un desbalance para el Perú, que exporta al país asiático principalmente minerales, productos pesqueros y agroindustriales e importa maquinaria y equipo, automotores, así como textiles y confecciones.

Los desafíos de las relaciones económicas y comerciales entre China y América Latina son varios, pero el principal es darle un contenido cualitativo más equitativo, evitando la reprimarización de las economías de la región; promoviendo la asociación estratégica de empresas y gobiernos; y generando condiciones de incentivo a la innovación y desarrollo tecnológico mutuamente conveniente.

\section{REFERENCIAS BIBLIOGRÁFICAS}

Adam Smith (1776). La riqueza de las naciones (C. Rodríguez Braun, Trad.). Titivillus. https:// www.memoriapoliticad emexico.org/Textos/1Independencia/Imag/1776-AS-LRN.pdf

Aviles Quintanar, D. A. y Wong González, P. (2019). China y el efecto de reprimarización en América Latina. 3C Empresa. Investigación y pensamiento crítico, 8(3), 118-149. doi: http://dx. doi.org/10.17993/3cemp.2019.080339.118-149

Banco Mundial. (2021). World Integrated Trade Solution; Perú Exportación. Proporción de Asociados (\% por país de 1992 al 2018). https:// wits.worldbank.org/CountryProfile/es/Country/PER/StartYear/1992/EndYear/2018/TradeFlow/ExportPartener/BY-COUNTRY/Indicator/XPRT-PRTNR-SHR

Bartesaghi, I. (2019). Los desafíos de China en América Latina. Working Paper Series (WPS) de Red China y América Latina: Enfoques Multidisciplinarios (REDCAEM), (9). http://chinayamericalatina.com/wp-content/uploads/2019/04/ WP9-Mar-2019-REDCAEM-2.pdf

BBC News Mundo. (08 de junio de 2020). "La gente no confía en EE.UU. porque no es fiel a sus ideales" / Reflexiones sobre la pandemia [Archivo de Vídeo]. Youtube. https://youtu.be/h8F5qz$70 \mathrm{jWk}$

Durán Lima, J. y Pellandra, A. (2017). La irrupción de China y su impacto sobre la estructura productiva y comercial de América Latina y el Caribe. CEPAL, Serie Comercio Internacional, N 131. https://repositorio.cepal.org/bitstream/handle/11362/41021/1/S1700028_es.pdf

Enrique Cornejo Digital. (12 de octubre de 2020). La Gran Guerra Comercial. Entrevista al economista Oscar Ugarteche [Archivo de Vídeo]. Youtube. https://youtu.be/K2CmiLy8usg

Fernández Arce, A. (2008). China: el asombro, crónicas y reportajes. Ediciones COPÉ, PetroPerú.

Fortune. (2020). Global 500. https://fortune.com/ global500/2020/search

Kissinger, H. A. (1994). La Diplomacia (M. Utrilla, Trad.). Ediciones B, S.A. https://filosofiadelaguerra.files.wordpress.com/2018/04/ kissinger-la-diplomacia.pdf

Krugman, P. R., Obstfeld, M. y Melitz, M. J. (2012). Economía Internacional: Teoría y Política (9a ed.). Pearson Educación, S.A.

López Villafañe, V. (2018). Las Relaciones Económicas de China con Latinoamérica. Bolsa de Comercio de Rosario, Revista Institucional, 
(1534). https://www.bcr.com.ar/es/print/pdf/ node/36882

Xi Jinping. (08 de enero de 2015). Inaugura la Primera Reunión Ministerial del Foro China-CELAC en Beijing. Presidente Xi Jinping pronuncia discurso [Comentario en la página web Ministerio de Relaciones Exteriores de la República Popular China]. https://www.fmprc.gov.cn/esp/ $\operatorname{zxxx} / \mathrm{t} 1227481 . \mathrm{shtml}$ 